http://doi.org/10.15359/ree.12-2.9

\title{
LA DIVERSIDAD EN LOS PROCESOS EDUCATIVOS: BASES PARA UNA PROPUESTA CONCEPTUAL
}

\author{
Dora Hernández Vargas ${ }^{1}$ \\ Docente de la División de Educación Básica (CIDE)-Universidad Nacional \\ Heredia, Costa Rica
}

"No sé si me gusta más de ti lo que te diferencia de mí o lo que tenemos en común.

Te guste o no, me caes bien por ambas cosas.

Lo común me reconforta, lo distinto me estimula".

Joan Manuel Serrat

Recibido: 7 de mayo, 2007 • Aprobado: 12 de junio, 2007

Resumen: La tesis principal de este trabajo es que lo que hace problemático el abordaje del concepto de diversidad son las consecuencias que derivemos de su reconocimiento. $Y$ este reconocimiento se hace desde la pedagogía. Luego de examinar, de forma sucinta, la trascendencia de la idea de diversidad en las principales tradiciones de la filosofía social, la autora propone las bases conceptuales que, desde estas lecturas ideológicas deben dirigir el abordaje pedagógico del concepto.

Palabras clave: Diversidad, exclusión, inclusión, currículum para la diversidad, pedagogía para la diversidad.

Abstract: The main thesis of this work is that what makes problematic the diversity concept approach are the consequences that we derive from its recognition. And this recognition comes from pedagogy. After examining, briefly, the importance of the idea of diversity in the main traditions of the social philosophy, the author proposes the conceptual bases that, from these ideological readings must direct the pedagogical boarding of the concept.

Key words: Diversity, exclusion, inclusion, curriculum for diversity, pedagogy for diversity.

Máster en pedagogía con énfasis en Diversidad en los Procesos Educativos. Experiencia en Educación Musical y profesora de la División de Educación Básica. Centro de Investigación y Docencia en Educación, Universidad Nacional. dorahv@gmail.com 


\section{Introducción}

Como estudiante de la Maestría en Pedagogía con énfasis en diversidad en los procesos educativos de la Universidad Nacional, solía cuestionarme los referentes desde los que analizábamos el concepto de diversidad. En primer lugar, cabía preguntarse qué debe entenderse por este concepto. ¿Se trataba de un concepto unívoco? O, por el contrario, ¿se trataba de un término que admite múltiples interpretaciones? En segundo lugar, estaba consciente de que el abordaje de la diversidad que interesaba era el que tenía trascendencia pedagógica $y$, entonces, las preguntas eran: ¿podemos concebir a todos los alumnos como diversos, o sólo a aquellos que se salen de la norma?, ¿qué aspectos del currículum deben ser transformados frente a la consideración de la diversidad?, ¿es posible atender a la diversidad a partir de un currículum común, o necesitamos de tantos currículos como culturas o personas atendamos pedagógicamente?

Es a partir de estas inquietudes que decidí investigar sobre el concepto de diversidad en los procesos educativos, construido en la Maestría. Luego de encontrar que había consenso, por parte de algunos, y contradicciones, por parte de otros, en torno al abordaje del concepto, me di a la tarea de definir mis bases conceptuales, que podrían servir de insumo para la construcción de una propuesta del concepto, que partiera de una visión pedagógica de la diversidad. Con este fin, creí necesario hacer una revisión de la trascendencia de la idea de diversidad en las principales tradiciones de la filosofía social.

Ahora, cabe preguntarse acerca del interés que comporta dicha revisión. Para empezar, es evidente el hecho de que entre los seres humanos existen diferencias, algunas veces de matiz $y$, en otras ocasiones, de peso. Entonces, ¿en dónde radica la problematicidad del concepto de diversidad, si es que hay alguna?

A mi modo de ver, esta problematicidad reside en su trascendencia o significación. Es decir, que lo que hace problemático el abordaje del concepto de diversidad son las consecuencias que derivemos de su reconocimiento. Por supuesto, que en lo que concierne a este trabajo, lo que interesa es la trascendencia que tiene el reconocimiento de la diversidad en los procesos educativos, y de éstos en su vínculo con la sociedad. De manera que resulta 
conveniente dar una mirada a las principales visiones que, sobre la sociedad, nos aporta la filosofía.

Esto deja entrever que el abordaje del concepto de diversidad se hace necesario, desde una concepción de sociedad. El currículum no es neutral. Bien anotaba Freire que la educación es un acto político, por lo que el abordaje de la diversidad no deja de serlo.

En el análisis de la diversidad en los procesos educativos, se hace necesario evaluar la fuerza transformadora del proyecto educativo, o, en dado caso, de los proyectos educativos. Reconocer la escuela como una institución que participa en forma sustantiva en la construcción de la ciudadanía, resulta imperativo, así como estudiar de manera sistemática sus formas de participación en este proceso.

\section{Idea de la diversidad en las tradiciones de la filosofía social}

Para analizar lo que significa el concepto de diversidad en las principales tradiciones de la filosofía social, seguiremos la tipología adoptada por Sacristán (2002), quien identifica tres principales tradiciones de pensamiento que denomina: a. Liberal democrática, $b$. Comunitarismo y c. Republicanismo democrático.

\section{Idea de la diversidad en el pensamiento liberal democrático}

Es evidente para muchos que no existe un solo liberalismo, sino que bajo este nombre encuentran abrigo múltiples corrientes ideológicas, que van desde los capitalistas liberales de la escuela de los Chicago Boys, hasta el racionalismo crítico de los teóricos de la Sociedad abierta, firmes defensores del individuo frente a los oligopolios del capital.

Pero, a pesar de las diferencias, los liberalismos comparten ciertos valores comunes, a saber: la prioridad del individuo frente a la colectividad, la igualdad formal entre los seres 
humanos, el universalismo, la neutralidad de las instituciones ante las diferentes creencias, la tolerancia ante la diversidad de ellas y la confianza en el carácter perfectible de las instituciones (Gray, citado por Sacristán, 2002).

Desde la óptica liberal, la diversidad es consecuencia de los procesos de individuación. La individualidad se asume como la cualidad y la posibilidad de diferenciarse de los demás, admitiendo la libertad y la autonomía de cada uno y su reconocimiento en las otras personas, requisito esencial para vivir en sociedad.

Ante la pregunta: ¿cuál es la esencia del individuo para el liberalismo?, éste responde que es la de aparecer individualizado a priori como una condición, metafísicamente esencial, que le viene dada al margen del individuo. Las cualidades de ese individuo abstracto y universal son independientes de su origen, de su condición personal o de su procedencia (etnia, género, clase social, etc.), porque constituyen la base de una identidad esencial para los sujetos, concebidos como libres, autónomos, con capacidad de elegir (Sacristán, 2002).

Precisamente, esta posibilidad del ente humano de elegir su ser y su devenir, es la base para que el pluralismo y la democracia liberales sean posibles. Efectivamente, de forma correlativa al derecho a la diferencia, que es inherente a todo individuo, los otros, principalmente el Estado, se hallan constreñidos por un deber de respetar y tolerar la diversidad que marca al individuo. Sacristán (2002) considera que la postura liberal ante el pluralismo (incluido el cultural) es tolerante e igualitaria; que no es beligerante en la promoción de una opción o cultura determinadas y que en la idea de ser una forma de vida y de expresión, corresponde a las opciones privadas tomar ese tipo de decisiones.

De acuerdo con este paradigma de hacer política, el Estado ha renunciado a la potestad de imponer a los hombres un determinado proyecto de felicidad y, sobre todo, de un ideal antropológico. Y, con esta renuncia, ha abdicado, también, a promover un modelo de vida entre los individuos. Ahora bien, no obstante la neutralidad que debe caracterizar al Estado liberal, su política admite cierto asimilacionismo cultural, pues el Estado no puede existir sin un marco común que abarque a todos los individuos. Esto, según el mismo Sacristán (2002), a pesar de que la pedagogía en el liberalismo se ha apoyado en cuatro pilares básicos: la construcción y la 
defensa del individuo moral, la propagación de la racionalidad científica, la tolerancia hacia los otros y la legitimación de las instituciones sociales.

El peligro que se corre en el abordaje de la diversidad desde esta corriente de pensamiento, yace en concebirla desde la perspectiva de la sola diferencia individual. Esto, por cierto, puede llevarnos a la atomización del currículum. De esta manera, se asumiría como deseable la existencia de un currículum para cada persona, adecuado a sus necesidades e intereses particulares. Si fuera así, ¿dónde quedarían los lazos comunes y cómo podríamos tener un proyecto de sociedad? Sólo sería posible construir proyectos de individuo, pero no de sociedad.

Desde esta perspectiva, además, se corre el riesgo de olvidar las diferencias entre colectivos. Al enfatizar el abordaje de la diversidad desde la diferencia individual, olvidamos las diferencias culturales y lingüísticas, por ejemplo. Suponemos que las diferencias son naturales y que la diversidad es un fenómeno natural, que se da de por sí, dejando de lado un elemento central de este análisis, a saber: que la diferencia es problemática porque las relaciones de poder han hecho que las diferencias se conviertan en exclusiones. Sobre este punto se discutirá más adelante.

\section{Idea de la diversidad en el pensamiento comunitarista}

De igual forma que se ha hecho la observación respecto del liberalismo, bajo la denominación comunitarismo, se comprenden múltiples corrientes de pensamiento, desde opciones políticas caracterizadas por el conservadurismo hasta formas populistas de hacer política.

Sin embargo, más allá de las disidencias, lo que para nuestros efectos interesa de los comunitaristas, y que vendría a fungir como factor común, es su posición ante la diversidad y el llamado hecho cultural.

Sobre este particular, las tesis comunitaristas asumen la cultura como "elemento aglutinador de los grupos humanos, a la vez que establece la diferencia entre ellos por ese 
motivo" (Sacristán 2002, p. 181). Aquí estriba la diferencia sustancial que existe entre liberalismo y comunitarismo. Si para el liberalismo la diversidad es una consecuencia de las opciones que los individuos adoptan como suyas; para el comunitarismo, la diversidad es una condición del ser humano que viene dada por la cultura en que el individuo ha nacido. Dicho de otra forma, lo que marca la diferencia, desde la perspectiva comunitarista, no reside en las decisiones que tome el individuo, sino en la pertenencia de esa persona a un determinado grupo cultural, el llamado hecho cultural.

Como podemos ver, para las corrientes comunitaristas, la diversidad es un tema que debe ser abordado desde la perspectiva del multiculturalismo. Este punto, tiene implicaciones de gran significación en el campo educativo, pues, como dice Sacristán (2002), la multiculturalidad, como variedad cultural de hecho, es innegable, y, además, es un proyecto, una ideología y una cosmovisión.

Como parte de esta cosmovisión, el multiculturalismo comunitarista sostiene que debe reconocerse la existencia de comunidades distintas, lo cual planteará conflictos a la educación cuando éstas convivan dentro de un mismo marco político y territorial. En primer lugar, las posiciones más conservadoras del comunistarismo aspiran a constituir comunidades cerradas en sus tradiciones $\mathrm{y}$, en consecuencia, con sus exclusivas instancias educativas. Otras derivaciones, igualmente conservadoras, optarán por otorgarle a la familia derechos exclusivos sobre la educación de sus hijos, con lo que se llega a extremos tales como reivindicar el derecho de los padres a decidir si renuncian al sistema escolar público, y optar por educar a sus hijos en casa, con un currículum doméstico, en el estricto sentido de la palabra.

Asimismo, podemos identificar dentro del multiculturalismo comunitarista posiciones que Sacristán (2002) califica como relativismos perspectivistas, que, en suma, se caracterizan porque no admiten que las prácticas y las creencias de una cultura puedan entenderse desde otra. Este perspectivismo considera que no es posible construir un currículum común abierto, que vaya más allá de las fronteras culturales de cada comunidad. En estas tesituras, se exige la desarticulación de lo que se podría llamar política educativa estatal, y se reclama el derecho de las comunidades culturales a articular un proyecto curricular propio. Desde estos presupuestos, según Sacristán (2002): 
(...) la escuela tenderá a organizarse como una microcomunidad que sirve a la comunidad en la que se inserta, de acuerdo con los valores de ésta, restaurándolos y afianzándolos, brindando a los individuos referencias para la adquisición de una identidad diáfana apoyada en los valores morales y en la cultura de dicha comunidad. Los profesores deberán ser "afiliados" a los valores de dicha comunidad, respetuosos guardianes y propagadores de los mismos (p. 189).

Es decir, que será una escuela cuyo objetivo principal es preservar y mantener la pureza de la cultura, por lo que podría darse el peligro de que, al reclamar la diferencia cultural para una comunidad respecto de otras, se niegue, después, la diversidad y se dé la discrepancia hacia los ciudadanos libres y autónomos.

\section{Idea de la diversidad en el republicanismo cívico}

Del comunitarismo es importante destacar que, a pesar de los peligros que plantea, nos recuerda que el individuo existe y vive dentro de un contexto social y no como entidad abstracta e impersonal, por lo que, aparte de la búsqueda de su libertad, debe procurar construir lazos de solidaridad con los otros que le rodean.

No obstante, para las tesis que Sacristán (2002) denomina republicanistas, la cultura no es la única forma de integración social posible, pues más allá del hecho cultural, es factible, según este autor, la integración política referida a la civitas, al Estado, que es de carácter más abstracto y que abarca a todos los ciudadanos, asegurando la lealtad a una 'cultura política' compartida. Esta idea de la integración política se sostiene sobre la convicción de que es posible aspirar al bien común, como un valor absoluto y universal.

Dentro de una democracia, la posibilidad de esta comunidad política en modo alguno pasa por la aniquilación de las diferencias, en cuenta las diversidades culturales de sus ciudadanos, pero se afirma que más allá de nuestra pluralidad se debe articular un proyecto compartido de sociedad equitativa y libre. Dentro de este contexto, el Estado se percibe a sí mismo como neutral, pero se conceptúa a la sociedad como plural, es decir, que dentro de un 
mismo marco político común, pueden convivir una pluralidad de grupos humanos cultural, lingüística y etnológicamente diversos.

Ahora bien, a pesar de que este ideal de neutralidad política, asegure a los individuos el respeto a su particularidad cultural, también protege la libertad de esos mismos individuos de optar por valores y costumbres aun diferentes de las que, en principio, caracterizan a su comunidad. Por supuesto que el respeto a la pluralidad cultural y a la libertad individual, deben darse dentro de los límites que exige la construcción de un proyecto moral cívico y político común.

No se trata de negar a la diversidad su valor, sino, sencillamente, de asumirlo en su justa dimensión, pues frente al derecho a la diferencia, debe contraponerse la necesidad de la coexistencia y de la interdependencia, para hacer posible el diálogo, lo cual conlleva un mínimo universalismo ético, de procedimientos de comunicación y de significados que, sin duda, debe ser pluralista.

Lo anterior tiene implicaciones de trascendencia para la educación, pues de un extremo debe ser fiel reflejo de una sociedad abierta y, como afirma Sacristán (2002), permitir a los individuos la toma de decisiones acerca de la cultura a la que se puede acceder, para que el individuo pueda automodelar su subjetividad, al margen y sin el control total de la familia, la iglesia o la escuela.

Por otro lado, esta misma educación debe ser promotora de opciones, de modelos de ser humano y de sociedad, que se plasmen en el currículum y que sea éste una selección valorada de la cultura. Por consiguiente, aunque el sistema educativo debe ser lo suficientemente flexible como para incluir el variopinto acervo de diferencias culturales, o de otro tipo, que coexisten en la sociedad política, debe ir más allá, posicionando al sujeto en una situación en la que sea capaz de hacer uso de su libertad de pensamiento para que, mediante el ejercicio de la crítica, reconstruya su entorno, siempre en busca de la edificación de una sociedad más justa, libre y equitativa. Al respecto, Sacristán (2002) expresa que la escuela es un aparato de reproducción inevitable y necesario de la sociedad y de la cultura dominantes, pero que, también, puede ser y ha sido un instrumento de reconstrucción de ambas, si cuenta con un proyecto adecuado y lo desarrolla de manera coherente. 
A pesar de que reconocer la diversidad responde a una necesidad profundamente humana, no podemos deducir que de este reconocimiento deba existir una educación diferente para grupos y sujetos distintos. Lo mismo que en busca de un proyecto común, tampoco podemos revalidar la teoría y la práctica homogenizadora de la educación moderna. Al respecto, Sacristán (2002) considera que: “El currículum común derivado del supuesto ilustrado de que existe y es conveniente una cierta universalidad, es compatible con el derecho a un currículum diferente, que también se deriva del pensamiento ilustrado. En cada aula, en cada centro escolar y en todo el sistema educativo hay que dejar espacio para diferentes opciones culturales, para una diferenciación que no sea discriminatoria para nadie" (p. 245).

En definitiva, para las tesis republicanistas, el currículum común debe ser el correlato de las sociedades modernas, caracterizadas por ser complejas, cambiantes y por ser el resultado de intensos intercambios culturales que las torna híbridas. Ahora, a pesar de que, en efecto, se valora el hecho de que el currículum es culturalmente relevante, un currículum para la diversidad debe ir más allá de la cultura, facultando al ser humano para la crítica de su entorno, y facilitándole el desarrollo de las competencias necesarias para construir un proyecto de sociedad que no sólo le asegure su libertad individual a ser diferente, sino que se entronque dentro de un marco de solidaridad con sus semejantes.

\section{La relevancia política del concepto de diversidad y la vocación política de la educación para la diversidad}

Frente a esta toma de postura en torno al modelo social que puede asumirse como deseable, podemos decir que el concepto de diversidad, en modo alguno, es una idea unívoca, mucho menos inocente. No solamente existen múltiples formas de comprender y de asumir la diversidad, sino que, también, la concepción que adoptemos responderá a una determinada visión ideológica de la comunidad política. 
El concepto de diversidad, desde una óptica crítica, redefine la diferencia, entendamos la relevante, como un producto del poder. Sobre este particular, Bhabha (citado por Giroux, 2001) escribe que:

\begin{abstract}
La idea de que la diversidad cultural constituye un problema, porque ya hay muchas culturas diferentes no es la razón por la que existe la diferencia cultural. La diferencia cultural es un discurso elaborado en un momento en el que se están desafiando elementos del poder o la autoridad (...). La diferencia cultural no resulta difícil, si se quiere, por el hecho de que haya diferentes culturas, sino porque existe una cuestión particular sobre la redistribución de los bienes entre las culturas o los fondos de las culturas (...) (p. 71).
\end{abstract}

De allí, que un discurso que admita la relevancia política de la diversidad, deba ser, esencialmente, reivindicativo ante la desigualdad impuesta por las relaciones y estructuras del poder social.

Otro tanto cabe decir respecto de la tentativa de elaborar una pedagogía o, como generalmente se le conoce, una educación para la diversidad. No es posible admitir el cariz reivindicativo del concepto de diversidad, sin que esto repercuta en una redefinición de la misión de la tarea educativa. Así, el propósito de la educación deberá ubicarse más allá de la transmisión de conocimientos, para reposicionarse dentro de la labor de reconstrucción de los significados sociales en torno a las diferencias.

Entonces, el educador, revalorado y apoderado como agente del cambio social, asumirá por tarea, de acuerdo con McLaren (1997), el cuestionar la ubicación, la posicionalidad y la especificidad del conocimiento (en términos de ubicación de raza, clase y género de los estudiantes), y de generar una pluralidad de verdades, al mismo tiempo que sitúa la construcción de significados en términos de intereses materiales, los que funcionan en la construcción de efectos de verdad, es decir, en la producción de formas de inteligibilidad y de prácticas sociales.

Una educación para la diversidad, pues, no se agota en la aspiración, establecida en la Declaración de Jomtien (1990) a pesar de la nobleza de la idea, de garantizar a cada persona la posibilidad real de acceder y sobrevivir dentro del sistema educativo. Es claro que una educación para la diversidad debe hacer suya la preocupación de Jomtien, por suprimir las 
barreras educativas que puedan impedir el aprovechamiento de las oportunidades escolares de los llamados grupos desasistidos, entre los cuales se cuentan: colectivos en situación de pobreza, niños de la calle, niños trabajadores de zona rural, nómadas, migrantes, indígenas y población perteneciente a minorías.

Sin embargo, la educación para la diversidad debe ir más allá, y constituirse en un instrumento para la formación de una ciudadanía crítica que sepa leer su condición diversa, y plantear soluciones para acceder a cada vez mayores niveles de equidad. Sobre este particular, cabe destacar la idea de Giroux (2001) de que la llamada educación política permite a los alumnos, expresarse de manera crítica y modificar la estructura participativa y el horizonte de debate en el que se construyen sus identidades, los valores y los deseos. Una educación política asienta los parámetros pedagógicos que permiten a los estudiantes entender cómo influye el poder en sus vidas, cómo influyen ellos en el poder y de qué manera pueden utilizarlo, para consolidar y ampliar su papel de ciudadanos críticos.

De esta forma, el ideal antropológico de un currículum para la diversidad deberá contemplar un ciudadano con las competencias cognitivas y epistemológicas suficientes para revisar, críticamente, su identidad, comprendiendo las relaciones de poder y dominación que han contribuido a su determinación, dotándola de nuevos significados y valoraciones. Asimismo, este ciudadano deberá tener las competencias emocionales e intelectuales para comprender la identidad del otro, su situación histórica y política, así como plantear nuevas fórmulas de equidad y convivencia en relación con ese otro.

\section{Lo problemático de la diversidad para la pedagogía}

En algún momento, la doctrina educativa ha definido el reto de la diversidad como la posibilidad de acceso al currículum de todo el alumnado, aceptando las diferencias en todos los niveles, para procurar dar respuestas educativas justas y acordes con las necesidades planteadas. El reconocimiento de las diversidades existentes constituye el punto de partida 
para evitar que las diferencias se conviertan en desigualdades y desventajas entre los individuos (Belmonte, 1998).

Esta forma de asumir el desafío de la diversidad tiene consecuencias importantes. Por un lado, permite reconocer distintos referentes de diversidad, incluso más allá del hecho cultural, entre ellos: capacidad, estilos de aprendizaje, ritmos de aprendizaje y motivaciones. En el otro extremo, esta forma de entender la diversidad exigirá una revisión estratégica (metodológica) de la forma de atender la diversidad. De tal manera que se conciben, al menos, dos grandes líneas de acción, según Belmonte (1998):

- $\quad$ Elaboración de estrategias diferenciadoras que traten de compartir y de respetar las diferencias, promoviéndolas cuando sea preciso, y

- Elaboración homogeneizadora y centrípeta, que pretenderá compensar y superar las desigualdades.

En este planteamiento, el objetivo de la educación para la diversidad es la pedagogía centrada en el que aprende, así como en la formación del que enseña, con capacidad para no generar segregaciones institucionales (Devalle y Vega, 1999).

Así pues, planteado en estos términos, el reto de la diversidad es el desafío de permitir el acceso de todas las personas al currículum. Pero, ¿cuáles son las consecuencias del reconocimiento de la diversidad como un concepto dinámico, político, una identidad que nace de las relaciones asimétricas de poder?

La respuesta es clara. Una educación consciente de la realidad injusta que ha dado pie a la construcción de las llamadas identidades diversas, es decir, las identidades construidas sobre un factor de diferencia, debe ser capaz de cuestionar las asunciones políticas y las relaciones de determinación sobre las que se fundan las verdades sociales, tanto en las comunidades en las que éstas funcionan como en la sociedad, en general, de la que son una parte (McLaren, 1997).

Se trata, pues, de una pedagogía de la solidaridad que permita a los individuos repensar los significados sobre los cuales se ha construido su identidad, ya sea su identidad no diversa como su identidad diversa, para diseñar nuevos lazos de solidaridad social, y, por tanto, 
reelaborar sus identidades. El fin último de esta pedagogía es la formación de una ciudadanía crítica, una ciudadanía "pluralizada e hibridada", como lo enuncia McLaren (1997), que pueda reconocer, con valor e inteligencia, la necesidad de enmendar las injusticias que subyacen en la formación de las identidades diversas, para construir nuevos significantes que sirvan para elaborar nuevas relaciones y estructuras de poder, mucho más equitativas.

Esto significa que una educación para la diversidad está más allá de un cambio estratégico o metodológico, y que exige una reelaboración del currículum, empezando por una revisión de los fundamentos antropológicos y sociales que le sustentan, modificando objetivos y contenidos, no para crear políticas de diferencia que colapsan en nuevas formas de separatismo (McLaren, 1997), sino para diseñar una política educativa que permita dar a luz una nueva ciudadanía que aspire a la libertad de la opresión y la exclusión sociales.

\section{Bases para una propuesta conceptual}

Si se retoma lo dicho en la sección anterior, es evidente el hecho de que entre los seres humanos existen muchas diferencias, por lo que bien puede decirse que, la disparidad es una condición inherente a nuestra naturaleza humana. Sin embargo, también es notorio que así como pueden hallarse factores que nos diferencian, se encuentran elementos que nos asemejan y nos unen. Igualmente, es importante subrayar que no toda diferencia resulta problemática. Habrá diferencias, como las que median entre zurdos y diestros que, en el momento actual, no plantean ningún problema significativo (Hernández, 2005).

Por supuesto, también debe reconocerse que el concepto de diversidad se caracteriza por su naturaleza relativa y abierta. Se trata de un concepto que cambia de acuerdo con las condiciones históricas. Con el ejemplo que recién expusimos, si bien es cierto, actualmente, la diferencia entre zurdos y diestros no hace mella, hasta hace algunas décadas ser zurdo constituía un problema muy serio, particularmente, dentro de los procesos educativos. 
Por consiguiente, a efectos de delimitar las bases para una propuesta conceptual, debemos decir que no interesan todas las diversidades, sino solamente aquellas, respecto de las cuales, debido a la estructura y las relaciones del poder imperante, son causa de exclusión.

A pesar de que no pueden manejarse los conceptos de diversidad y de exclusión como si fuesen sinónimos, la diversidad que realmente es problemática, es aquella asociada a fenómenos de exclusión. El problema de la diversidad radica en el hecho de que, debido al carácter centralizador y homogenizador del poder político en nuestras sociedades, las comunidades o colectivos diversos (en relación con la identidad central o nacional) han sido excluidos de los procesos políticos, incluyendo los procesos educativos.

En relación con los procesos educativos, cabe matizar en el sentido de que la exclusión de la diversidad tiene dos modalidades importantes:

a. Cuando a determinado colectivo o comunidad se le obstaculiza, o, en el peor de los casos, se le niega el acceso a la educación.

b. Cuando determinado colectivo o comunidad es excluido del currículum, pues su identidad no se encuentra representada dentro de él $y$, por el contrario, se enfrenta al avasallamiento por parte del sistema educativo que promueve, activamente, la identidad oficial.

Así las cosas, y en lo que concierne a la pedagogía para la diversidad, ésta debería atender un reclamo histórico de los colectivos y las comunidades diversas, para ser incluidos dentro del currículum y, en general, dentro de los procesos educativos.

Con base en este análisis del concepto de diversidad desde la pedagogía, podríamos esbozar algunas características propias de un currículum para la diversidad, como materialización de esta nueva pedagogía propuesta. 


\section{Un currículum para una ciudadanía democrática}

Es necesario reconocer que la articulación de una política educativa de atención a la diversidad, apareja el grave riesgo de la fragmentación curricular. Efectivamente, la atención a la diversidad, no debe enclaustrar a los colectivos y a las comunidades diferentes, en guetos educativos, con currículos cerrados y, especialmente, diseñados para ellos.

De acuerdo con Savater (2002), lo que realmente nos amenaza hoy es, de nuevo, la recurrencia al origen como condicionamiento inexorable de la forma de pensar: dividir el mundo en guetos estancos y estancados de índole intelectual. Es decir, que sólo los nacionales puedan comprender a los de su nación, que sólo los negros puedan entender a los negros, los amarillos a los amarillos y los blancos a los blancos, que sólo los cristianos comprendan a los cristianos y los musulmanes a los musulmanes, que sólo las mujeres entiendan a las mujeres, los homosexuales a los homosexuales y los heterosexuales a los heterosexuales. Que cada tribu deba permanecer cerrada sobre sí misma, idéntica según la identidad establecida por los patriarcas o caciques del grupo, ensimismada en su pureza de pacotilla. Y que, por tanto, debe haber una educación diferente para cada uno de estos grupos que los respete, es decir, que confirme sus prejuicios y no les permita abrirse y transformarse con los demás.

Por el contrario, en opinión de Hernández, Ibarra y Quesada (1993), un currículum para la diversidad tendrá que ser abierto y plural, que se caracterice, esencialmente, por abrir un espacio para todas las identidades, pero también por ofrecer la oportunidad de reconocer lo universal que nos une como humanos y como miembros de una comunidad política, valorando, como consecuencia, las relaciones y los intercambios entre los distintos colectivos. Por supuesto, ninguna de estas identidades debe atribuirse a sí misma, un lugar central en dicho currículum.

El currículum que se propone debe aspirar a la construcción de una identidad política (De Lucas, 2003), que conserve el valor de la identidad cultural sin pretender que éste monopolice la identidad democrática. Se trata de un currículum cuyo objetivo final sea la formación de una ciudadanía democrática, dentro de la cual se encuentren incluidas, en un plano de equidad, múltiples identidades. 
En consecuencia, dentro de este currículum, la integración y la inclusión, no se conciben como un proceso de asimilación, sino de integración político-democrática, lo cual es vital porque una pedagogía para la diversidad debe, también, promover contextos de convivencia, que permitan a distintas comunidades y colectivos construir un proyecto común dentro de un marco de pluralidad.

\section{Un currículum para la inclusión}

Conviene acotar que el currículum que se propone no debe ser uno que, sencillamente, admita la diversidad, sino que su proyecto debe orientarse hacia la diversidad, para la diversidad. Este tipo de educación para la diversidad no debe ser solamente una teoría, debe ser una práctica necesaria, pues no se trata de tolerar la diferencia, sino de valorar la diferencia como factor que incide sobre los procesos de aprendizaje (Devalle y Vega, 1998).

Tal y como lo proponen estos autores, para la construcción de una educación para la diversidad, es importante reconocer que la escuela no se enfrenta a la diversidad como si se encarara ante un accidente, sino que resulta imperativo que la escuela sea consciente de que la diversidad es una característica intrínseca del contexto socio cultural y político, dentro del cual se halla inmersa. Se trata de transformar el paradigma hasta ahora vigente, desde el cual la diferencia es vista como desventaja o como déficit, para constituirse en un valor que orienta la acción educativa hacia principios de igualdad, justicia y libertad.

Debe concluirse, parafraseando a Devalle y Vega (1998), que esta escuela para la diversidad es la propia de una sociedad democrática que ha incorporado dentro de sus proyectos de Estado el objetivo inclaudicable de garantizar a todos (viéndolos a todos como seres humanos diversos) el derecho a la educación. Es decir, que un currículum para la diversidad, no solamente debe procurar ofrecer espacio para las múltiples identidades que convivan dentro de un determinado espacio socio-político, sino que, además, debe procurar cumplir con la aspiración de garantizar acceso universal a la educación, removiendo, al efecto, 
los obstáculos que puedan existir para que determinadas identidades o colectivos puedan acceder a la educación.

\section{Un currículum para resignificar identidades}

Un currículum para la diversidad, a la par de ser abierto a las múltiples identidades presentes en la sociedad, debe tener también por objeto la resignificación de las identidades.

Si se desea que el currículum contribuya, de forma real, a la formación de una ciudadanía democrática, éste debe permitir una relectura crítica de las identidades diversas, así como de la llamada identidad oficial. Sobre este aspecto, la meta es abandonar cualquier idea sobre una identidad hegemónica y centralizadora, y, a la vez, reivindicar el derecho de las comunidades y de los colectivos a visibilizar sus identidades dentro del mismo proceso pedagógico, sin que esto implique una idealización de dichas identidades, sino desde una perspectiva crítica y reconstructiva.

Por supuesto, esta relectura de las múltiples identidades debe realizarse en un contexto de diálogo e intercambio entre colectivos. Es decir, el objetivo no es repetir el esencialismo monolítico de los centrismos, sino, precisamente, reconocer que el valor de nuestra identidad es correlativo a la valía del otro. McLaren (1997) opina que la necesidad de crear una política de alianza y de construcción, de soñar juntos, de mostrar una solidaridad que vaya más allá de la condescendencia ha de ser ganada con la lucha. Considera que no está centrada en torno a los imperativos del mercado, sino que se desarrolla a partir de los imperativos de liberación, de democracia y de ciudadanía crítica.

Así pues, el proceso de resignificación de las identidades ha de realizarse dentro de un marco de solidaridad y de comunidad, que permita reinterpretarnos, pero también construir un proyecto en común con las identidades. Un proyecto tal que se articule en torno a un concepto de ciudadanía híbrida, que lejos de asociarse a una identidad hegemónica -el católico, mestizo e hispanoparlante de nuestra Costa Rica-, esté en la condición de vincularse a un proyecto común que tenga por protagonistas todas las identidades del país. 


\section{Un currículum para la transformación de la realidad, hacia una realidad más justa}

Para concluir, es necesario enfatizar que el reto de la diversidad no se circunscribe, únicamente, a asegurar el derecho de las comunidades y de los colectivos diversos a la educación. Por esta razón, la respuesta pedagógica no debe circunscribirse, exclusivamente, a la individuación pedagógica. El reto de la diversidad tiene una fuerte connotación justiciera, y, en consecuencia, exige una profunda reforma curricular que afecte todos sus factores y elementos. Una reforma de tal naturaleza que transforme el currículum en un instrumento abierto a todas las identidades de los distintos colectivos y de las diversas comunidades de la sociedad, y que permita una relectura de dichas identidades, una relectura que, precisamente, sea liberadora de los condicionamientos impuestos por las asimetrías del poder imperante.

No obstante lo anterior, ésta es también una propuesta de solidaridad, que invita a los distintos sujetos a hacer esta relectura de su identidad, dentro de un espacio de diálogo y reflexividad, de modo que esta resignificación se realice de forma crítica y constructiva. En fin, se trata de una propuesta que tiene por objeto la formación de un nuevo concepto de ciudadano, un ciudadano que se reconozca a sí mismo como parte de determinado(s) colectivo(s) y que, a la vez, parta de un proyecto democrático y solidario.

\section{Unas últimas palabras}

Una de las principales tesis de este estudio es que el sistema educativo es un factor clave en la formación de identidades, de la misma forma que la formación del Estado nacional costarricense, en su época, sirvió para delinear y consolidar la nacionalidad costarricense, destruyendo cualquier otra identidad en el imaginario de nuestro país.

La propuesta conceptual que aquí se postula tiene por objeto reivindicar el currículum como espacio público, para repensar y reconstruir las identidades. Debemos recordar que lo diverso se constituye siempre en relación con algún otro referente: se es diverso "con respecto 
a". Pero hay que tener suficiente habilidad para discernir y suficiente sensibilidad para entender, cuáles diversidades nos complementan y nos enriquecen como personas y como comunidad, y cuáles diversidades nos excluyen y nos segregan. La labor pedagógica debería ayudarnos y exigirnos a desarrollar esas capacidades.

\section{Referencias}

Belmonte, M. (1998). Atención a la Diversidad. Diseño de estrategias y evaluación formativa basada en el análisis de tareas. Tomo I. Bilbao: Ediciones Mensajero.

De Lucas, J. (2003). Globalización e identidades. Claves políticas y jurídicas. Barcelona: Icaria Editorial.

Devalle, A. y Vega, V. (1999). Una escuela en y para la diversidad. El entramado de la diversidad. Buenos Aires: AIQUE.

Giroux, H. (2001). Cultura, política y práctica educativa. Barcelona: Graó.

Hernández, D. (2005). El concepto de diversidad en los procesos educativos presente en el Plan de estudios y en los programas de curso de la Maestría en Pedagogía con énfasis en diversidad en los procesos educativos de la Universidad Nacional. Trabajo final para los cursos Didáctica para la construcción del conocimiento y Análisis pedagógico de la diversidad. Maestría en Pedagogía con énfasis en diversidad en los procesos educativos. Heredia, Costa Rica: Universidad Nacional.

Hernández, O., Ibarra, E. y Quesada, J. R. (1993). Discriminación y racismo en la historia costarricense. San José: Editorial de la Universidad de Costa Rica.

McLaren, P. (1997). Pedagogía crítica y cultura depredadora. Políticas de oposición en la era posmoderna. Barcelona: Ediciones Paidós.

Sacristán, J. G. (2002). Educar y convivir en la cultura global. Madrid: Editorial Morata.

Savater, F. (2002). El valor de educar. Barcelona: Ariel. 